\title{
Characterization of Roasted Coffee Aroma by Optimizing Roaster Parameters
}

\author{
FIRMAN RIDWAN* \& RONI NOVISON \\ Department of Mechanical Engineering, Faculty of Engineering, University of Andalas, Padang, Indonesia \\ *Corresponding author: firmanridwan@ft.unand.ac.id
}

\begin{abstract}
This study was aimed to improve the quality of aroma of roasted coffee by using Taguchi's design as experimental technique. The roaster parameters evaluated were temperature, incubation time, moisture content and cylinder rotational speed. An orthogonal array $\mathrm{L}_{9}$, signal to noise ratio and ANOVA were employed to investigate the influence of the roaster parameters. The results showed that the optimal roasted coffee aroma was produced at a temperature of $170^{\circ} \mathrm{C}$, incubation time of 14 minutes, moisture content of $6 \%(\mathrm{v} / \mathrm{w})$ and cylinder rotational speed of $50 \mathrm{rpm}$. The most to less significant roasting parameters as observed in this study were as follows: temperature, incubation time, moisture content and cylinder rotational speed. Furthermore, the results showed that the Taguchi design was better than the full experimental design in solving experiments with a minimum number.
\end{abstract}

Keywords: ANOVA, Coffee roaster, Roasted coffee aroma, Signal to noise ratio, Taguchi technique

Copyright: This is an open access article distributed under the terms of the CC-BY-NC-SA (Creative Commons Attribution-NonCommercial-ShareAlike 4.0 International License) which permits unrestricted use, distribution, and reproduction in any medium, for non-commercial purposes, provided the original work of the author(s) is properly cited.

\section{INTRODUCTION}

The insight of coffee roaster is growing rapidly along with the growing interest in drinking coffee. Many beverage stores provide coffee as a main menu. Generally, coffee drinks are commercialised using Arabica, Robusta or a mixture of both. Coffee lovers prefer to have brewed coffee immediately after roasting. The high number of coffee drink lovers is due to multiple factors. Two of the major factors are the flavor and typical aroma of the coffee (Kumazawa \& Masuda, 2003).

In general, consumers do not have preference for green coffee beans in term of their flavor and taste. The aroma and flavor of coffee are highly dependent on roasting. Roasting is a process that determines the physiochemical characteristics of coffee beans such as flavor and taste. Sunarharum et al. (2014) provide an overview of the relationship between the flavor component and the sensory properties expressed in the complex coffee matrix. Gabriel-Guzmán et al. (2017) proposed a methodology for investigating changes in surface image of coffee beans during roasting based on the Rescaled range fractal analysis. The analytical results showed that physical changes in coffee beans such as fracture and color change can be utilised for indirect detection of roasting level through the fractal index.
Roasting is carried out in order to obtain the organoleptic properties (taste, color and unique structure) and volatile compounds (aldehydes, ketones, acetic acid, and formic acid) (Illy, 1998; Hernández et al., 2007), which represent the desired trait of the coffee. During roasting, coffee beans undergo a reaction that results in changes in physical properties (color, volume and density) and chemical composition (release of $\mathrm{CO}_{2}$, evaporation of water and volatile compounds) (Illy, 1998; Kumazawa \& Masuda, 2003). Thus, the composition of volatile and other compounds in green coffee beans is important in determining the characteristics of coffee aroma. Raising the temperature up to $100^{\circ} \mathrm{C}$ caused the green coffee beans to undergo structural changes, such as reduction of humidity level (moisture loss) from $8-12 \%$ to $5 \%$ (Illy, 1998). After roasting, the color of coffee beans change from green to yellow with increasing level of fragility of the coffee beans' structure (Hernández et al., 2007). Overheating causes coffee beans to be bitter and less fragrant. On the other hand, insufficient roasting times is reported to be responsible for the unfavorable organoleptic characteristics (overall taste, color and structure) (Lyman et al., 2003; Yeretzian et al., 2002).

Coffee roasting is usually performed at different temperatures and times depending on 
the desired characteristics. The roasting temperature can be detected by the color of the resulting roasted coffee beans, the loss of weight and variation of flavor and aroma caused by the changes in the chemical composition that occur during the roasting process (Hernández et al., 2007). Marija et al. (2012) conducted a study to evaluate changes in the characteristics of the resulting coffee beans as total weight loss, breakage and discoloration. According to their report, coffee was grouped into two, namely Outspan and Guaxupe. Their study also included the results of coffee beans for Outspan and Guaxupe based on the value of $\mathrm{L}^{*}$ (color parameters) at the end of roasting. In addition, the mean values of bean's breaking force was generated at 37.82 Newton and 35.39 Newton for Outspan and Guaxupe, respectively.

The chemical composition and volatile compounds in coffee are produced in thermal reactions during the roasting process. Monoterpenes is one of the compounds that persist when the coffee is burned (Del Terra et al., 2013). Lee et al. (2016) in their research on the coffee aroma during the fermentations of green coffee beans evaluated the growth of Rhizopus oligosporus when grown on volatile and nonvolatile green coffee. The results showed that there is variation in the aroma of coffee through the fermentation of green coffee beans.

Furthermore, roasting coffee requires precise controls and innovative techniques. Rosa et al. (2016) monitored the change of the chemical markers appearance on the surface of the bean during the roasting process by using mass spectrometry. The authors used a non-target and non-volatile approach with ambient techniques (EASI) integrated into a single quadrupole mass analyzer to detect the changing of chemical compounds during the roasting of coffee bean.

In this study, an effort has been made to estimate the best quality of roasted coffee aroma using experimental data. Quality planning on roasting was arranged at the beginning of the process by adjusting the factors that influence the coffee flavor such as temperature, time, moisture, and cylinder rotational speed. Taguchi technique was used to identify the significance of the influence of each factor to the aroma of roasted coffee. The choice of Taguchi method was due to its efficiency that requires small amount of trials compared to the other experimental designs
(Genichi, 1989; Ross, 1996). The aroma model was then developed by response surface methodology.

\section{MATERIALS AND METHODS}

\section{Taguchi technique}

In this research, Taguchi technique was used to optimize the roaster parameter for improving the quality of roasted coffee aroma. This method is appropriate and effective to overcome the response with a minimum number of experiments that deal with a number of variables (Ross, 1996).

The Taguchi technique is a statistical technique developed by Genichi Taguchi to improve the quality of products through experimental design. In this technique, orthogonal array is utilised to arrange the experiment with a special set of matrices. This standard matrix aims to establish the minimum amount of experiment and include as much information as possible from all significant factors of roaster parameters. The selection of input level combinations is the most important step for each experiment. Taguchi method uses signal to noise ratio (SNR) to evaluate the quality of a product. Characteristics of the quality is the result of a process related to quality. The characteristics of quality measured by Taguchi technique can be divided into three categories as follows:

a. smaller-the-better (STB)

STB is used when the smaller the calculation value the better the product quality.

$$
\begin{aligned}
& S T B=-10 \log \left(\frac{1}{n} \sum_{i=1}^{n} y_{i}^{2}\right) \\
& n=\text { number of experiments } \\
& y=\text { measured value }
\end{aligned}
$$

b. $\quad$ larger-the-better (LTB)

LTB is used when the greater the calculation value the better the product quality.

$$
L T B=-10 \log \left(\frac{1}{n} \sum_{i=1}^{n} \frac{1}{y_{i}^{2}}\right)
$$

c. normal-the-better (NTB)

NTB is used when closer to the calculation value, the better the product quality.

$$
\begin{aligned}
& N T B=-10 \log \left(\frac{\bar{y}}{S_{y}^{2}}\right) \\
& S_{y}^{2}=\text { the variation of } y
\end{aligned}
$$


The Taguchi study has three stages involved from the entire experimental approach. These three stages are planning, implementation and analysis. The planning stage provides information on the set of experiments, in which all selected parameters of coffee roaster are varied over a specified range while the implementation stage is a part of the process of taking data processing coffee roasting. Finally, the analysis step is the last important step to determine the desired significant data.

\section{Roasting experiments design using Taguchi method}

The coffee roaster machine is made of stainless steel with a thickness of $2 \mathrm{~mm}$, a diameter of 300 $\mathrm{mm}$ and a length of $300 \mathrm{~mm}$ (see Figure 1). This machine consists of two cylinders: the rotor cylinder and the stator cylinder. Direct current motor is used to drive the rotor cylinder. At the planning stage, the parameters of the roaster to be selected were specified and named as factors. These factors were temperature, time, moisture content and rotational speed of the rotor cylinder. Each factor has three levels. At the implementation stage, nine experiments using various combination of roasting parameters were performed. This study generated the design of experiments using Orthogonal Array $\mathrm{L}_{9}$ contained in the Taguchi method that was based on the degrees of freedom, factors and factor levels as shown in Table 1.
The roasting processes aims to reach the best quality of the aroma and flavor of the coffee beans with heat treatment. To do so, three assesments were chosen to test the taste of roasted coffee for each combination. Figure 2 shows the results of coffee beans with various combination of roaster parameters for nine experiments. The type of coffee beans used in this study was Arabica, originated from Payakumbuh, West Sumatra. The volume used was $100 \mathrm{~g}$ of coffee beans per experiment.

Table 1. Orthogonal Array L.

\begin{tabular}{ccccc}
\hline Number of & \multicolumn{4}{c}{ Factor } \\
\cline { 2 - 5 } experiment & $\mathrm{A}$ & $\mathrm{B}$ & $\mathrm{C}$ & $\mathrm{D}$ \\
\hline 1 & 1 & 1 & 1 & 1 \\
2 & 1 & 2 & 2 & 2 \\
3 & 1 & 3 & 3 & 3 \\
4 & 2 & 1 & 2 & 3 \\
5 & 2 & 2 & 3 & 1 \\
6 & 2 & 3 & 1 & 2 \\
7 & 3 & 1 & 3 & 2 \\
8 & 3 & 2 & 1 & 3 \\
9 & 3 & 3 & 2 & 1 \\
\hline
\end{tabular}

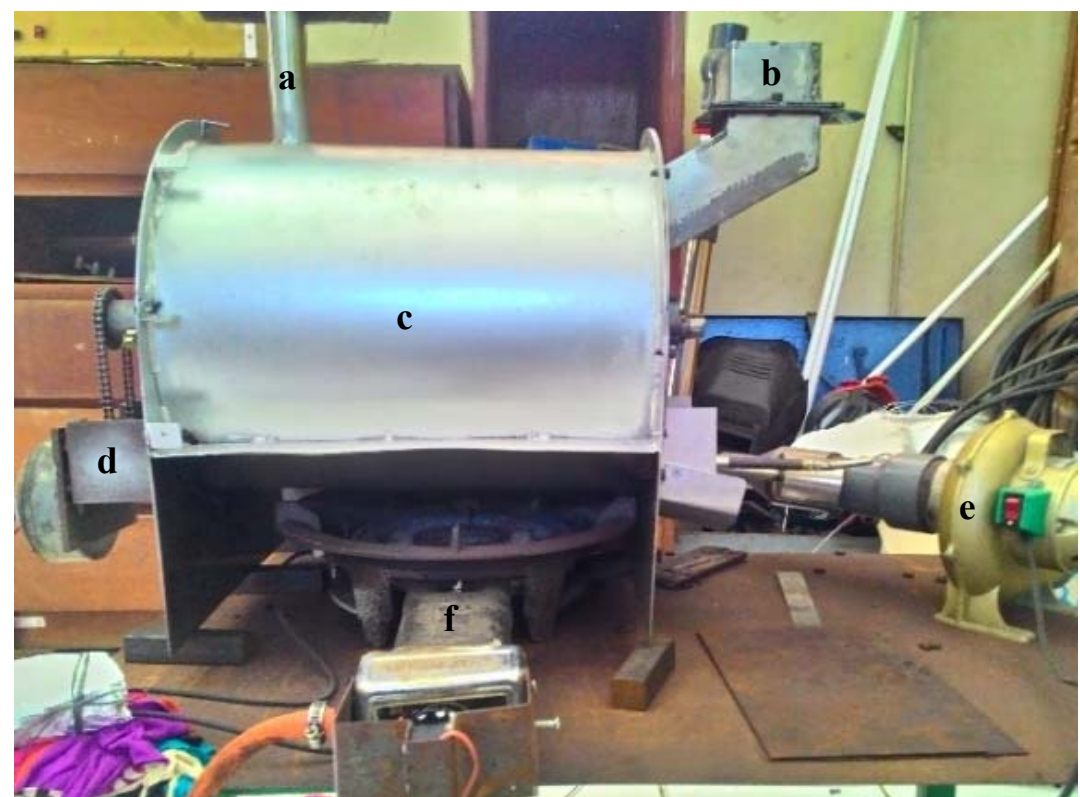

Figure 1. Coffee roaster machine: (a) exhaust-pipe; (b) hopper; (c) coffee roaster; (d) actuator; (e) blower; and (f) burner. 


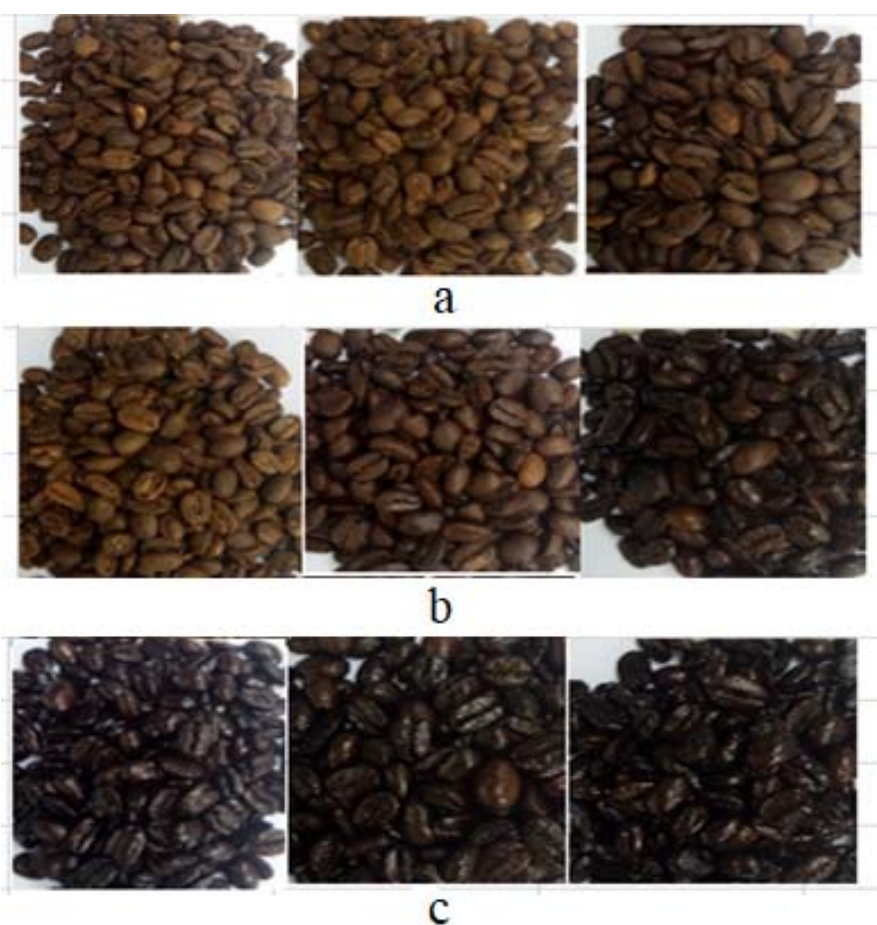

Figure 2. Roasted coffee beans produced from experiments with various combination of parameters: (a) level 1; (b) level 2; and (c) level 3.

The response variable and independent variables used in this study are as follows:

1. Variable response is the coffee aroma level.

2. Independent variable consists of four factors, which are suspected to affect the quality of roasted coffee. Each factor has three levels. The specification of the factors and their levels are shown in Table 2.

Table 2. Specification of factors and their levels.

\begin{tabular}{lcccc}
\hline \multicolumn{1}{c}{ Factor } & Variable & 1 & 2 & 3 \\
\hline Temperature $\left({ }^{\circ} \mathrm{C}\right)$ & $\mathrm{A}$ & 160 & 170 & 180 \\
Time $(\mathrm{s})$ & $\mathrm{B}$ & 10 & 12 & 14 \\
Moisture $(\%)$ & $\mathrm{C}$ & 6 & 7 & 8 \\
Speed $(\mathrm{rpm})$ & $\mathrm{D}$ & 40 & 50 & 60 \\
\hline
\end{tabular}

Based on the number of level and factor of roaster parameters, orthogonal array matrix $L_{9}$ was chosen with the number of trials as much as nine times.

\section{RESULTS \& DISCUSSION}

The experimental data analysis was divided into three parts. Firstly, we calculated the response of the average value. The results describe the order of influence of each factor from the largest to the smallest as shown in Table 4. Secondly, we calculated the response of SNR values. These results describe the quality of coffee were the largest value in the table represents the best quality of coffee as shown in Table 5. The factors that generated the greatest response were selected as the proposed design for the type of quality character according to LTB principle. Thirdly, we calculated the response of ANOVA.

Based on the SNR calculation in Table 3, the best roasted coffee aroma was produced at a temperature of $170^{\circ} \mathrm{C}$, time of 14 minutes, moisture content of $6 \%(\mathrm{v} / \mathrm{w})$ and cylinder rotational speed of $50 \mathrm{rpm}$. These can be seen from the results of the average calculation with a value of 4 and SNR with a value in 12.04.

Table 4 shows the mean values of each response on each factor. The mean values on each factor were calculated by subtracting the largest average value from the smallest average value of its factor. Figure 3 shows the roasted coffee characteristic of each factor that we considered. The most to less significant roaster parameters were temperature, incubation time, moisture content and cylinder rotational speed. The mean values of each factor were 1.67 (temperature) in the first rank, 1.00 (incubation time) in the second rank, 0.66 (moisture content) in the third rank and 0.44 (cylinder rotational speed) in the fourth rank. 
Table 3. Results of experiment data, average calculation and signal to noise ratio (SNR).

\begin{tabular}{|c|c|c|c|c|c|c|c|c|c|}
\hline \multirow{2}{*}{$\begin{array}{l}\text { Number of } \\
\text { experiment }\end{array}$} & \multicolumn{4}{|c|}{ Factor } & \multicolumn{3}{|c|}{ Assessment* } & \multirow[t]{2}{*}{$\bar{y}$} & \multirow{2}{*}{$\begin{array}{l}\text { SNR } \\
\text { Value }\end{array}$} \\
\hline & A & $\mathrm{B}$ & $\mathrm{C}$ & $\mathrm{D}$ & 1 & 2 & 3 & & \\
\hline 1 & 160 & 10 & 6 & 40 & $1^{*}$ & 1 & 1 & 1.00 & 0 \\
\hline 2 & 160 & 12 & 7 & 50 & $2^{*}$ & 1 & 2 & 1.67 & 3.01 \\
\hline 3 & 160 & 14 & 8 & 60 & $3^{*}$ & 3 & 2 & 2.67 & 8.03 \\
\hline 4 & 170 & 10 & 7 & 60 & 2 & 3 & 3 & 2.67 & 8.03 \\
\hline 5 & 170 & 12 & 8 & 40 & 3 & 4 & 4 & 3.67 & 11.04 \\
\hline 6 & 170 & 14 & 6 & 50 & $4^{*}$ & 4 & 4 & 4.00 & 12.04 \\
\hline 7 & 180 & 10 & 8 & 50 & 3 & 3 & 3 & 3.00 & 9.54 \\
\hline 8 & 180 & 12 & 6 & 60 & 4 & 3 & 4 & 3.67 & 11.04 \\
\hline 9 & 180 & 14 & 7 & 40 & 3 & 3 & 3 & 3.00 & 9.54 \\
\hline
\end{tabular}

*Note: Very fragrant: 4, Fragrant: 3, Simply fragrant: 2, Less fragrant: 1

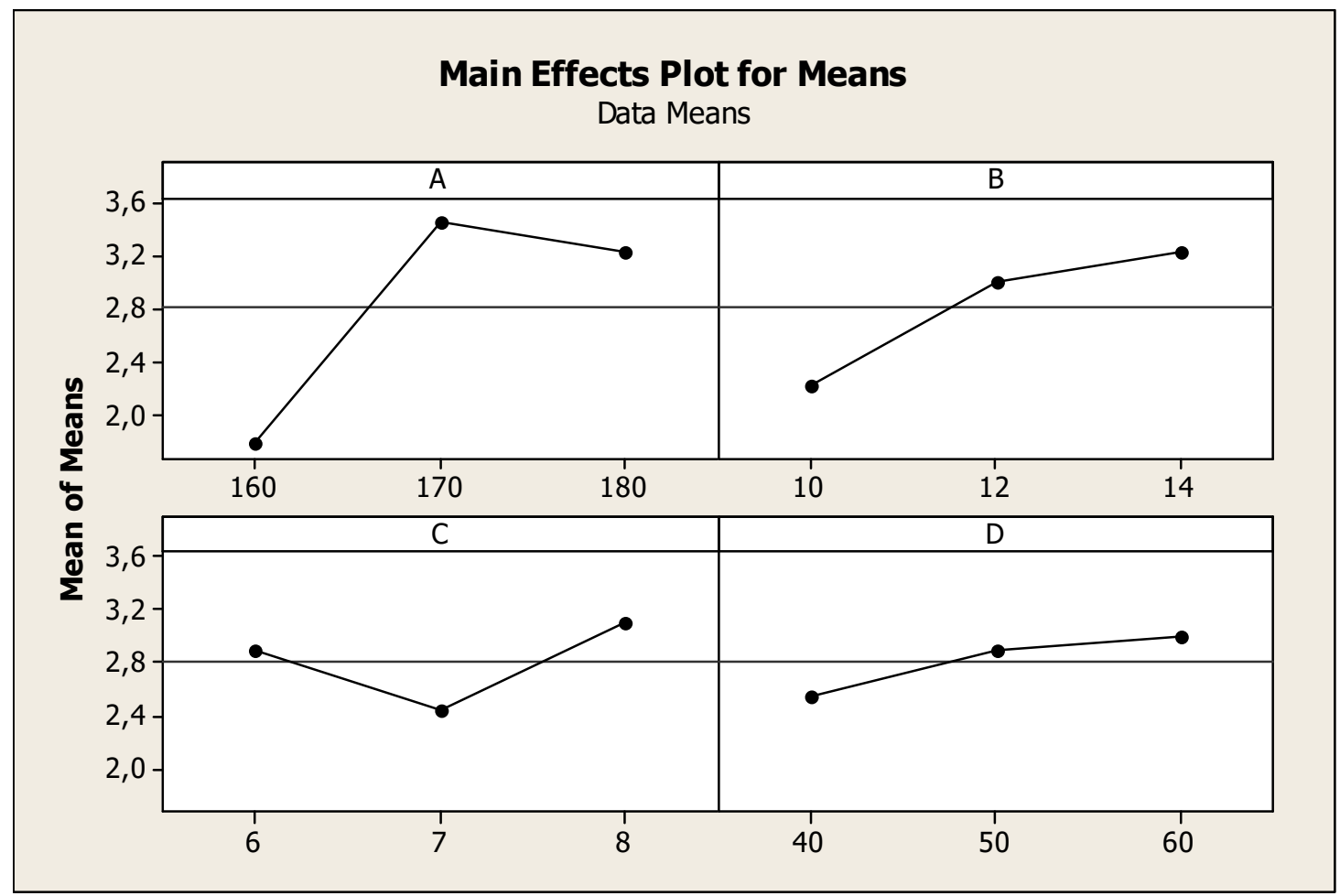

Figure 3. Coffee characteristic for each level of factor: (A) factor A; (B) factor B; (C) factor C; and (D) factor D.

Table 4. Response of average values.

\begin{tabular}{lcccc}
\hline Level & A & B & C & D \\
\hline 1 & 1.778 & 2.222 & 2.889 & 2.556 \\
2 & 3.444 & 3.000 & 2.444 & 2.889 \\
3 & 3.222 & 3.222 & 3.111 & 3.000 \\
Delta & 1.667 & 1.000 & 0.667 & 0.444 \\
Rank & 1 & 2 & 3 & 4 \\
\hline
\end{tabular}

Table 5 shows the response value of the SNR for each factor that we considered. The SNR values on each factor was calculated by subtracting the largest SNR value from the smallest SNR value. Figure 4 shows the response graph of the SNR to the roasting coffee aroma of each factor. It can be seen that the most to less significant roaster parameters as observed in this study were temperature, incubation time, moisture content and cylinder rotational speed. The SNR value of each factor is the percentage of temperature with the value of 6.69 in the first rank, incubation time with the value of 4.01 in the second rank, moisture content with the value of 2.68 in the third rank and the cylinder rotational speed with the value of 2.17 in the fourth rank.

Table 6 shows the results of ANOVA at significant level of $5 \%$. Based on Table 6 , we may conclude that all the factors that we considered had significant effect on the coffee bean characteristics. Factor A showed the most obvious influence on the quality of roasted coffee. 


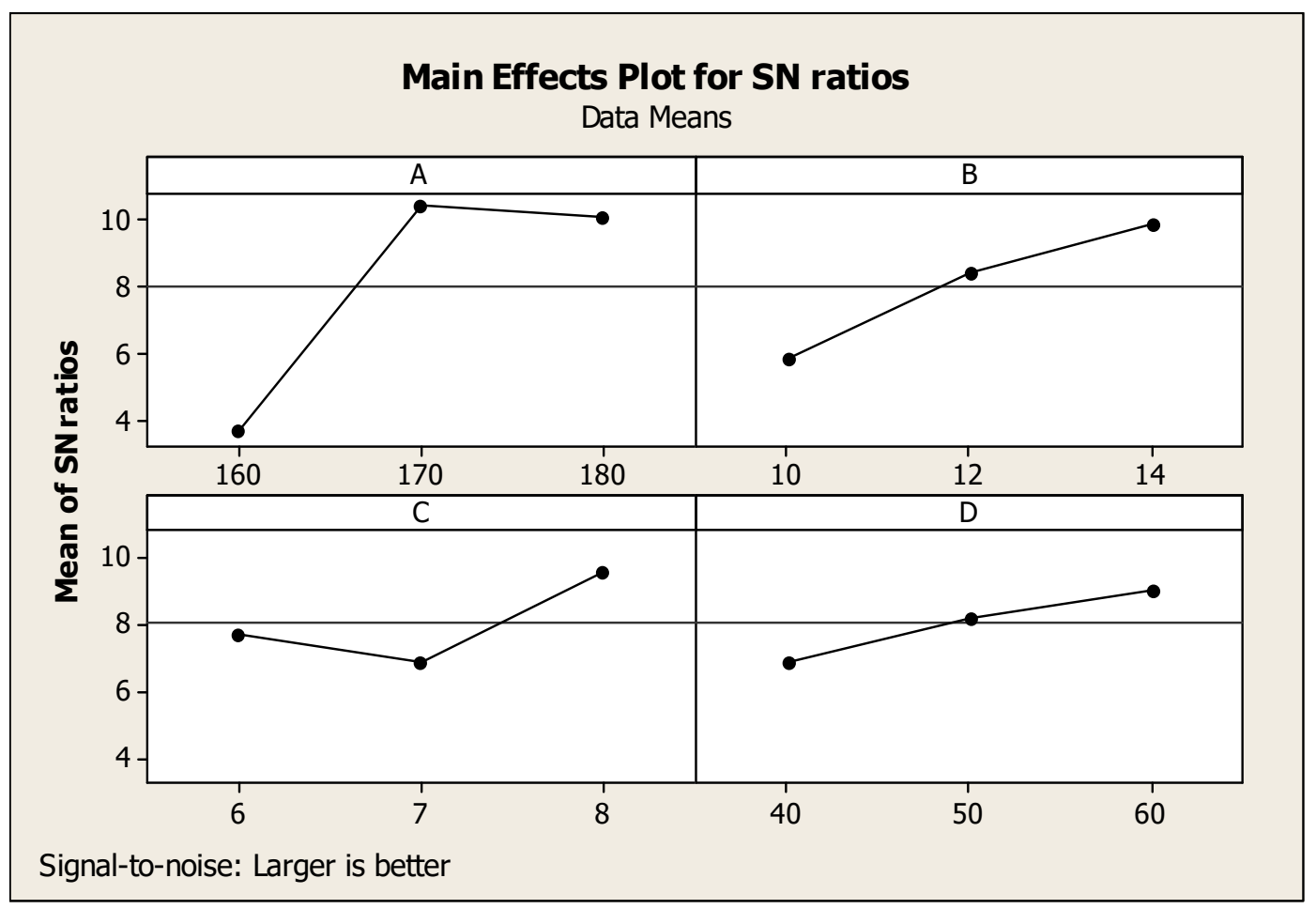

Figure 4. Signal to noise ratios of each response on each factor: (A) factor A; (B) factor B; (C) factor C; and (D) factor D.

Table 6. Analysis of variance (ANOVA).

\begin{tabular}{lccccc}
\hline $\begin{array}{l}\text { Source of } \\
\text { Variance }\end{array}$ & $\begin{array}{c}\text { Degree of } \\
\text { Freedom }(\mathrm{DF})\end{array}$ & $\begin{array}{c}\text { Sum Square } \\
(\mathrm{SS})\end{array}$ & $\begin{array}{c}\text { Mean Square } \\
(\mathrm{MS})\end{array}$ & F-ratio & Contribution (\%) \\
\hline Factor A & 2 & 15.4386 & 7.7193 & 116.9065 & 57.1028 \\
Factor B & 2 & 5.6724 & 2.8362 & 42.9534 & 20.6688 \\
Factor C & 2 & 2.7853 & 1.3926 & 21.0912 & 9.8982 \\
Factor D & 2 & 1.7204 & 0.8602 & 13.0275 & 5.9255 \\
Error & 18 & 1.1885 & 0.0660 & & 6.4046 \\
\hline Total & 26 & 26.8053 & 1.0310 & & 100 \\
\hline
\end{tabular}

Table 7 shows the uniformity of the proposed design for the roasted coffee bean characteristics. The least contributing factors influencing the coffee characteristics were temperature, followed by time in the second rank, moisture content in the third rank and rotation speed in the fourth rank. The percentage of contribution for all the factors were $57.1 \%$ (temperature), 20.7\% (time), 9.9\% (moisture content) and 5.9\% (rotation speed). These were also supported by the sum of squares values that represent the measure of variation or deviation from the mean.
The calculation of the total sum of squares considers both the sum of squares from the factors and randomness or error.

Table 7. Ranking based on influences of each factor.

\begin{tabular}{cccc}
\hline Ranking & Mean & SNR & ANOVA \\
\hline 1 & A & A & A \\
2 & B & B & B \\
3 & C & C & C \\
4 & D & D & D \\
\hline
\end{tabular}




\section{CONCLUSION}

Characterisation of coffee bean was conducted by roasting the coffee bean with various parameters of roaster through design of experiment using Orthogonal Array L9 specified by Taguchi method. Roasted coffee was assessed by three volunteers of coffee lover. Three responses values, response of mean value, response of SNR value and response of ANOVA were analysed. Based on the experiments, the optimal roasted coffee aroma was produced at a temperature of $170^{\circ} \mathrm{C}$, incubation time of 14 minutes, moisture content of $6 \%(\mathrm{v} / \mathrm{w})$ and a cylinder rotational speed of $50 \mathrm{rpm}$. All three responses, response of mean value, response of SNR value and response of ANOVA agreed that temperature was the most important contributing factors to the coffee bean characteristics with the value of response of 1.67 , $6.69,57.10 \%$ respectively. This was followed by time at the second rank with the response values of mean, SNR and ANOVA of 1.00, 4.01, $20.67 \%$, respectively, moisture content at the third rank with the response values $0.67,2.68$, $9.89 \%$, respectively and rotational speed of rotor cylinder at the fourth rank with the response values of $0.44,2.17,5.90 \%$, respectively.

\section{ACKNOWLEDGEMENTS}

This research was supported by Engineering Faculty of Andalas University with the grant contract no. DIPA: 15/UN.16.17.D/PL/2017.

\section{REFERENCES}

Del Terra, L., Lonzarich, V., Asquini, E., Navarini, L., Graziosi, G., Suggi Liverani, F. \& Pallavicini, A. (2013). Functional characterization of three Coffea arabica L. monoterpene synthases: Insights into the enzymatic machinery of coffee aroma. Phytochemistry, 89: 6-14.

Gabriel-Guzmán, M., Rivera, V.M., CocotleRonzón, Y., García-Díaz, S. \& HernandezMartinez, E. (2017). Fractality in coffee bean surface for roasting process. Chaos, Solitons \& Fractals, 99: 79-84.
Genichi, T. (1989). Quality Engineering in Production Systems. Singapore: McGraw-Hill Book Company.

Hernández, J. A., Heyd, B., Irles, C., Valdovinos, B. \& Trystram, G. (2007). Analysis of the heat and mass transfer during coffee batch roasting. Journal of Food Engineering, 78(4): 11411148.

Illy, A. V. R. (1998). Espresso coffee. San Diego: Academic Press.

Kumazawa, K. \& Masuda, H. (2003). Investigation of the change in the flavor of a coffee drink during heat processing. Journal of Agricultural and Food Chemistry, 51(9): 2674-2678.

Lee, L.W., Cheong, M.W., Curran, P., Yu, B. \& Liu, S.Q. (2016). Modulation of coffee aroma via the fermentation of green coffee beans with Rhizopus oligosporus: II. Effects of different roast levels. Food Chemistry, 211: 925-936.

Lyman, D.J., Benck, R., Dell, S., Merle, S. \& Murray-Wijelath, J. (2003). FTIR-ATR analysis of brewed coffee: effect of roasting conditions. Journal of Agricultural and Food Chemistry, 51(11): 3268-3272.

Marija R. J., Natalija. R.D., Biljana R.C., Slavica G. \& Bozana B. (2012). Changes of physical properties of coffee beans during roasting. Acta Periodica Technologica, 43: 21-31.

Rosa J.S., Freitas-Silva O., Rouws J.R.C., Moreira I.G.S., Novaes F.J.M., Azevedo D.A. \& Rezende C.M. (2016). Mass spectrometry screening of Arabica coffee roasting: A nontarget and non-volatile approach by EASI-MS and ESI-MS. Food Research International, 89 (Part 2): 967-975.

Ross, P. J. (1996). Taguchi Techniques for Quality Engineering: Loss Function, Orthogonal Experiments, Parameter and Tolerance Design: New York, NY: McGraw-Hill.

Sunarharum, W.B., Williams, D.J. \& Smyth, H.E. (2014). Complexity of coffee flavor: A compositional and sensory perspective. Food Research International, 62: 315-325.

Yeretzian, C., Jordan, A., Badoud, R. \& Lindinger, W. (2002). From the green bean to the cup of coffee: investigating coffee roasting by on-line monitoring of volatiles. European Food Research and Technology, 214(2): 92-104. 